\title{
Fuel gas operation management practices for reheating furnace in iron and steel industry
}

\author{
Chen, D.M. ${ }^{a, b}$, Liu, Y.H. ${ }^{a}$, He, S.F. ${ }^{c}$, Xu, S. ${ }^{c}$, Dai, F.Q. ${ }^{b}$, Lu, B. ${ }^{a,{ }^{*}}$ \\ ${ }^{a}$ School of Civil Engineering and Architecture, Anhui University of Technology, Ma'anshan, P.R. China \\ ${ }^{b}$ The State Key Laboratory of Refractories and Metallurgy, Wuhan University of Science and Technology, Wuhan, \\ P.R. China \\ 'Ma'anshan iron and Steel Co., Ltd, Ma'anshan, Anhui, P.R. China
}

\begin{abstract}
A B S T R A C T
How to evaluate the fuel gas operation (FGO) of various working groups (WGs) and working shifts (WSs) in reheating furnace is still ambiguous problem. In this paper, a nov elty time-s eries FGO evaluation model was proposed. The strategy mainly included: Firstly, the fuel gas per ton steel (FGTS) was calculated in certain time interval; Secondly, the FGTS time-s eries data set was formulated in statistical period; Thirdly, the FGTS time-series data set was divided according to working schedule; Lastly, the FGO evaluation model was established. Case study showed that: i) The fuel gas operation evaluation results of various WGs in different WSs were accorded with normal distribution; ii) For various WGs, A WG performed best, followed by C WG and D WG. The performance of B WG was the worst due to its violent fluctuation of fuel gas operation evaluation results in three WSs; iii) For different WSs, the day WS and swing WS performed well, whereas the performance of night WS was unsatisfactory. Discussion results showed that the improvement of working skills, working responsibility and working passion, which were effective measure to achieve energy saving in terms of operation, should be enhanced through skills training and the reward and punishment system. Generally, this novelty time-series FGO evaluation method could also be applied to other industrial equipment.
\end{abstract}

\begin{tabular}{l} 
A R T I C L E I N F O \\
\hline Keywords: \\
Iron industry; \\
Steel Industry; \\
Fuel gas operation (FGO) manage- \\
ment; \\
Reheating furnace; \\
FG0 evaluation model; \\
Fuel gas per ton steel (FGTS) time- \\
series; \\
Working groups; \\
Working shifts \\
*Corresponding author: \\
road_lu12@163.com \\
(Lu, B.) \\
Article history: \\
Received 26 March 2020 \\
Revised 9 July 2020 \\
Accepted 13 July 2020
\end{tabular}

(c) 2020 CPE, University of Maribor. All rights reserved.

\section{Reference}

[1] Duan, W., Yu, Q., Wang, K., Qin, Q., Hou, L., Yao, X., Wu, T. (2015). ASPEN plus simulation of coal integrated gasification combined blast furnace slag waste heat recovery system, Energy Conversion and Management, Vol. 100, 30-36, doi: 10.1016/i.enconm an.2015.04.066.

[2] Karali, N., Park, W.Y., McNeil, M. (2017). Modeling technological change and its impact on energy savings in the U.S. iron and steel sector, Applied Energy, Vol. 202, 447-458, doi: 10.1016/i.apenergy.2017.05.173.

[3] Chen, Q., Gu, Y., Tang, Z., Wei, W., Sun, Y. (2018). Assessment of low-carbon iron and steel production with $\mathrm{CO}_{2}$ recycling and utilization technologies: A case study in China, Applied Energy, Vol. 220, 192-207, doi: 10.1016/i. apenergy.2018.03.043.

[4] Lu, B., Tang, K., Chen, D., Han, Y., Wang, S., He, X., Chen, G. (2019). A novel approach for lean energy operation based on energy apportionment model in reheating furnace, Energy, Vol. 182, 1239-1249, doi: 10.1016/i.energy. 2019.06.076.

[5] Griffin, P.W., Hammond, G.P. (2019). Industrial energy use and carbon emissions reduction in the iron and steel sector: A UK perspective, Applied Energy, Vol. 249, 109-125, doi: 10.1016/i.apenergy.2019.04.148.

[6] Lu, B., Chen, G., Chen, D., Yu, W. (2016). An energy intensity optimization model for production system in iron and steel industry, Applied Thermal Engineering, Vol.100, 285-295, doi: 10.1016/i.applthermal eng.2016.01.064. 
[7] An, R., Yu, B., Li, R., Wei, Y.-W. (2018). Potential of energy saving and $\mathrm{CO}_{2}$ emission reduction in China's iron and steel industry, Applied Energy, Vol. 226, 862-880, doi: 10.1016/i.apenergy.2018.06.044.

[8] Chen, D., Lu, B., Zhang, X., Dai, F., Chen, G., Liu, Y. (2018). Fluctuation characteristic of billet region gas consumption in reheating furnace based on energy ap portionment model, Applied Thermal Engineering, Vol. 136, 152-160, doi: 10.1016/i.applthermal eng.2018.03.007.

[9] Chen, D., Lu, B., Chen, G., Yu, W. (2017). Influence of the production fluctuation on the proc ess energy intensity in iron and steel industry, Advances in Production Engineering \& Management, Vol. 12, No. 1, 75-87, doi: $10.14743 /$ apem2017.1.241.

[10] Zhang, Q., Zhao, X., Lu, H., Ni, T., Li, Y. (2017). Waste energy recovery and energy efficiency improvement in China's iron and steel industry, Applied Energy, Vol. 191, 502-520, doi: 10.1016/i.apen ergy.2017.01.072.

[11] Zhang, Q., Xu, J., Wang, Y., Hasanb eigi, A., Zhang, W., Lu, H., Arens, M. (2018). Comprehensive assessment of energy conservation and $\mathrm{CO}_{2}$ emissions mitigation in China's iron and steel industry based on dynamic material flows, Applied Energy, Vol. 209, 251-265, doi: 10.1016/i.apenergy.2017.10.084.

[12] He, K., Wang, L. (2017). A review of energy use and energy-efficient technologies for the iron and steel industry, Renewable and Sustainable Energy Reviews, Vol. 70, 1022-1039, doi: 10.1016/i.rser.2016.12.007.

[13] Peng, J., Xie, R., Lai, M. (2018). En ergy-related $\mathrm{CO}_{2}$ emissions in the China's iron and steel industry: A global supply chain analysis, Resources, Conservation and Recycling, Vol. 129, 392-401, doi: 10.1016/i.resconrec. 2016. $\underline{09.019}$.

[14] $\mathrm{Hu}, \mathrm{R} .$, Zhang, Q. (2015). Study of a low-carbon production strategy in the metallurgical industry in China, Energy, Vol. 90, Part 2, 1456-1467, doi: 10.1016/i.energy.2015.06.099.

[15] McBrien, M., Cabrera Serrenho, A., Allwood, J.M. (2016). Potential for en ergy savings by heat recovery in an integrated steel supply chain, Applied Thermal Engineering, Vol. 103, 592-606, doi: 10.1016/i.applthermal eng.2016. $\underline{04.099}$.

[16] Ke, H.-L., Dong, B., Ye, B. (2014). Research and application of slab heating curve in reheating furnace, Metallurgical Industry Automation, Vol. 38, No. 3, 50-55, doi: 10.3969/i.issn.1000-7059.2014.03.010.

[17] Mayr, B., Prieler, R., Demuth, M., Moderer, L., Hochenauer, C. (2017). CFD analysis of a pusher ty pe reheating furnace and the billet heating characteristic, Applied Thermal Engineering, Vol. 115, 986-994, doi: 10.1016/ j.applthermaleng.2017.01.028.

[18] Tang, G., Wu, B., Bai, D., Wang, Y., Bodnar, R., Zhou, C. (2018). CFD modeling and validation of a dy namic slab heating process in an industrial walking beam reheating furnace, Applied Thermal Engineering, Vol. 132, 779789, doi: 10.1016/i.applthermaleng.2018.01.017.

[19] Han, S.H., Chang, D., Kim, C.Y. (2010). A numerical analysis of slab heating characteristics in a walking beam type reheating furnace, International Journal of Heat and Mass Transfer, Vol. 53, No. 19-20, 3855-3861, doi: 10.1016/ j.i.iheatmasstransfer.2010.05.002.

[20] Emadi, A., Saboonchi, A., Taheri, M., Hassanpour, S. (2014). Heating characteristics of billet in a walking hearth type reheating furnace, Applied Thermal Engineering, Vol. 63, No. 1, 396-405, doi: 10.1016/i.applthermal eng. 2013.11.003.

[21] García, A.M., Amell, A.A. (2018). A numerical analysis of the effect of heat recovery burners on the heat transfer and billet heating characteristics in a walking-beam type reheating furnace, International Journal of Heat and Mass Transfer, Vol. 127, Part B, 1208-1222, doi: 10.1016/i.ijheatmasstransfer.2018.07.121.

[22] Han, S.H., Chang, D. (2012). Radiative slab heating analysis for various fuel gas compositions in an axial-fired reheating furnace, International Journal of Heat and Mass Transfer, Vol. 55, No. 15-16, 4029-4036, doi: $10.1016 /$ j.ijheatmasstransfer.2012.03.041.

[23] Han, S.H., Lee, Y.S., Cho, J.R., Lee, K.H. (2018). Efficiency analysis of air-fuel and oxy-fuel combustion in a reheating furnace, International Journal of Heat and Mass Transfer, Vol. 121, 1364-1370, doi: 10.1016 j.ijheatmasstransfer.2017.12.110.

[24] Wang, J.-G., Shen, T., Zhao, J.-H., Ma, S.-W., Wang, X.-F., Yao, Y., Chen, T. (2017). Soft-s ensing method for optimizing combustion efficiency of reheating furnaces, Journal of the Taiwan Institute of Chemical Engineers, Vol. 73, 112-122, doi: 10.1016/i.jtice.2016.09.037.

[25] Zhang, Q. (2017). Application of reheating furnace waste heat integrated utilization technology, Energy for Metallurgical Industry. Vol. 36, No. 1, 45-47, doi: 10.3969/i.issn.1001-1617.2017.01.011.

[26] Dal Magro, F., Jimenez-Arreola, M., Romagnoli, A. (2017). Improving energy recovery efficiency by retrofitting a PCM-based technology to an ORC system operating under thermal power fluctuations, Applied Energy, Vol. 208, 972-985, doi: 10.1016/i.apenergy.2017.09.054.

[27] Jiménez-Arreola, M., Wieland, C., Romagnoli, A. (2017). Response time characterization of organic rankine cycle evaporators for dynamic regime analysis with fluctuating load, Energy Procedia, Vol. 129, 427-434, doi: 10.1016 Li.egypro.2017.09.131.

[28] Pili, R., Romagnoli, A., Spliethoff, H., Wieland, C. (2017). Techno-economic analysis of waste heat recovery with ORC from fluctuating industrial sources, Energy Procedia, Vol.129, 503-510, doi: 10.1016/i.egypro.2017.09.170.

[29] Wang, L. (2016). An example of waste heat recovery of heating furnace bas ed on energy cascade utilization, Metallurgical Power, Vol. 1, 30-31, doi: 10.3969/i.issn.1006-6764.2016.01.010.

[30] Lu, B., Chen, D., Chen, G., Yu, W. (2017). An en ergy apportionment model for a reheating furnace in a hot rolling mill - A case study, Applied Thermal Engineering, Vol.112, 174-183, doi: 10.1016/i.applthermaleng.2016.10.080.

[31] Chen, D., Lu, B., Dai, G.-Q., Chen, G., Zhang, X. (2018). Bottl eneck of slab thermal efficiency in reheating furnace based on energy apportionment model, Energy, Vol. 150, 1058-1069, doi: 10.1016/i.energy.2018.02.149.

[32] Chen, D., Lu, B., Dai, F.-Q., Chen, G., Yu, W. (2018). Variations on billet gas consumption intensity of reheating furnace in different production states, Applied Thermal Engineering, Vol. 129, 1058-1067, doi: 10.1016/ j.applthermaleng.2017.10.096. 
[33] Ferreira, J.E.V., Pinheiro, M.T.S., dos Santos, W.R.S., da Silva Maia, R. (2016). Graphical representation of chemical periodicity of main elements through boxplot, Educación Química, Vol. 27, No. 3, 209-216, doi: 10.1016/ j.eq.2016.04.007.

[34] Hubert, M., Vandervieren, E. (2008). An adjusted boxplot for skewed distributions, Computational Statistics \& Data Analysis, Vol.52, No.12, 5186-5201, doi: 10.1016/i.csda.2007.11.008. 


\title{
Prakse ravnanja z zgorevalnim plinom za ogrevalne peči v železarski in jeklarski industriji
}

\author{
Chen, D.M. ${ }^{\mathrm{a}, \mathrm{b}}$, Liu, Y.H. ${ }^{\mathrm{a}}$, He, S.F. ${ }^{c}$, Xu, S. ${ }^{\mathrm{c}}$, Dai, F.Q. ${ }^{\mathrm{b}}$, Lu, B. ${ }^{\mathrm{a},{ }^{*}}$ \\ ${ }^{a}$ School of Civil Engineering and Architecture, Anhui University of Technology, Ma'anshan, P.R. China \\ ${ }^{b}$ The State Key Laboratory of Refractories and Metallurgy, Wuhan University of Science and Technology, Wuhan, \\ P.R. China \\ 'Ma'anshan iron and Steel Co., Ltd, Ma'anshan, Anhui, P.R. China
}

\section{POVZETEK}

Kako ovrednotiti upravljanje različnih delovnih skupin (WG) in delovnih izmen (WS) z zgorevalnim plinom (FGO) za ogrevalno peč je še vedno zahtevna naloga. $V$ tem prispevku je bil predlagan nov model ocenjevanja FGO s časovno vrsto. Strategija je v glavnem vključevala naslednje korake: 1) najprej je bil v določenem časovnem intervalu izračunan porabljen zgorevalni plin na tono jekla (FGTS), 2) nato je bil oblikovan nabor podatkov o časovni vrsti FGTS v statističnem obdobju, 3) nabor podatkov o časovni vrsti FGTS je bil razdeljen glede na delovni urnik in 4) bil je vzpostavljen model vrednotenja FGO. Študija primera je pokazala, da: i) so bili rezultati ocenjevanja upravljanja z zgorevalnim plinom različnih delovnih skupin v različnih delovnih izmenah usklajeni z normalno porazdelitvijo; ii) izmed različnih delovnih skupin se je najbolje odrezala delovna skupina A, sledili sta ji C in D. Učinkovitost delovne skupine $B$ je bila najslabša zaradi močnega nihanja rezultatov ocenjevanja upravljanja z zgorevalnim plinom v treh delovnih izmenah; iii) pri različnih delovnih izmenah sta se jutranja delovna izmena in popoldanska delovna izmena odrezali dobro, medtem ko je bil rezultat nočne delovne izmene nezadovoljiv. Rezultati razprave so pokazali, da je treba za izboljšanje delovnih veščin, delovne odgovornosti in delovne strasti, ki so učinkovit ukrep za doseganje varčevanja z energijo v smislu upravljanja, vpeljati sistem usposabljanja ter sistem nagrajevanja in kaznovanja. Na splošno bi lahko predlagano metodo ocenjevanja časovnih vrst FGO, ki je novost, uporabili tudi za drugo industrijsko opremo.

\section{PODATKI O ČLANKU}

Ključne besede:

Železarska industrija; Jeklarska industrija;

Upravljanje z zgorevalnim plinom (FGO);

Ogrevalna peč;

Model vrednotenja FGO;

Časovna vrsta zgorevalnega plina na tono jekla (FGTS);

Delovne skupine;

Delovne izmene

* Kontaktna oseba: road_lu12@163.com

(Lu, B.)

Zgodovina članka:

Prejet 26. marca 2020

Popravljen 9. julija 2020

Sprejet 13. julija 2020 